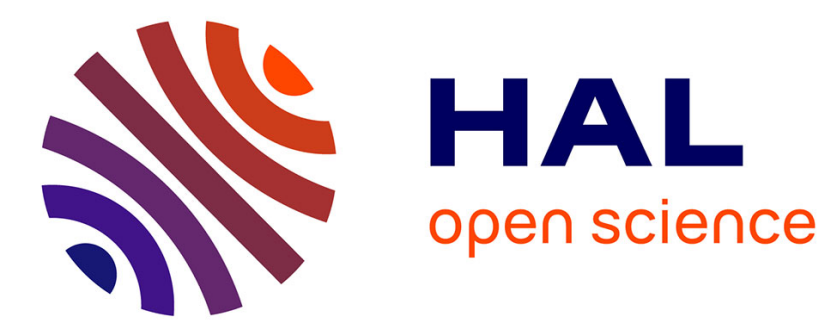

\title{
Mirrors used in the LIGO interferometers for first detection of gravitational waves
}

L Pinard, C Michel, B Sassolas, L Balzarini, J Degallaix, V Dolique, R

Flaminio, D Forest, M Granata, B Lagrange, et al.

\section{- To cite this version:}

L Pinard, C Michel, B Sassolas, L Balzarini, J Degallaix, et al.. Mirrors used in the LIGO interferometers for first detection of gravitational waves. Applied optics, 2017, 56 (4), 10.1364/AO.56.000C11 . in2p3-01452276

\section{HAL Id: in2p3-01452276 https://hal.in2p3.fr/in2p3-01452276}

Submitted on 2 Feb 2017

HAL is a multi-disciplinary open access archive for the deposit and dissemination of scientific research documents, whether they are published or not. The documents may come from teaching and research institutions in France or abroad, or from public or private research centers.
L'archive ouverte pluridisciplinaire HAL, est destinée au dépôt et à la diffusion de documents scientifiques de niveau recherche, publiés ou non, émanant des établissements d'enseignement et de recherche français ou étrangers, des laboratoires publics ou privés. 


\title{
The mirrors used in the LIGO interferometers for the first detection of gravitational waves
}

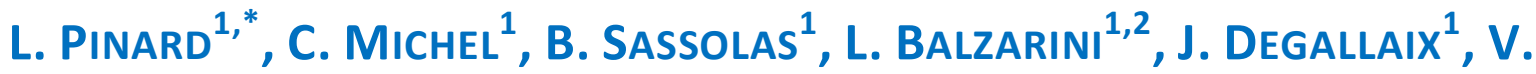 \\ Dolique $^{1}$, R. Flaminio ${ }^{3}$, D. Forest ${ }^{1}$, M. Granata ${ }^{1}$,B. Lagrange ${ }^{1}$, N. Straniero, \\ J. TEILLON ${ }^{1}$, G. CAGNOLI ${ }^{1,2}$ \\ ${ }^{1}$ Laboratoire des Matériaux Avancés - CNRS/IN2P3 - F-69622 - Villeurbanne France \\ ${ }^{2}$ Université Claude Bernard Lyon 1 - F-69622 - Villeurbanne France \\ ${ }^{3}$ National Astronomical Observatory of Japan, 181-8588 Tokyo, Japan \\ *Corresponding author: I.pinard@Ima.in2p3.fr
}

Received XX Month XXXX; revised XX Month, XXXX; accepted XX Month XXXX; posted XX Month XXXX (Doc. ID XXXXX); published XX Month XXX

\begin{abstract}
For the first time, a direct detection of gravitational wave occurred in the LIGO interferometers. These advanced detectors need large fused silica mirrors having optical and mechanical properties never reached up to now. This paper details the main achievements of these IBS coatings. O2016 Optical Society of America
\end{abstract}

OCIS codes: (310.1620) Interference coatings, (310.1860) Deposition and Fabrication; (310.4165) Multilayer design, (310.6860) Thin films, optical properties

http://dx.doi.org/10.1364/AO.99.099999

\section{INTRODUCTION}

On September 14, 2015 at 09:50:45 UTC, the two detectors of the Laser Interferometer Gravitational wave Observatory (LIGO) simultaneously observed a transient gravitational-wave signal [1]. The signal matches the waveform predicted by general relativity for the inspiral and merger of a pair of black holes and the ringdown of the resulting single black hole. These observations demonstrate the existence of binary stellar-mass black hole systems. This is the first direct detection of gravitational waves and the first observation of a binary black hole merger.

The Laboratoire des Matériaux Avancés (LMA) was in charge of providing the coatings for more than 30 large optics (34/35 cm up to $55 \mathrm{~cm}$ diameter and $20 \mathrm{~cm}$ thick) for Advanced LIGO and Advanced Virgo gravitational wave interferometers. In particular, LMA has coated the 20 arm cavity mirrors (Input Mirror (IM) and End Mirrors (EM)) of Advanced LIGO and all the mirrors of Advanced Virgo. Furthermore, we have coated the large Advanced Virgo Beam Splitter $(\varnothing 55 \mathrm{~cm}, 6.5 \mathrm{~cm}$ in Thickness) which is the largest optical part ever coated by Ion Beam Sputtering IBS) (Fig 1).

The mirror substrates are made in ultra-pure fused silica (Suprasil from Heraeus Quartz Glass) on which a Ti$\mathrm{Ta}_{2} \mathrm{O}_{5} / \mathrm{SiO}_{2}$ IBS multilayers is deposited [2]. The increased YAG input laser power in the interferometers implies also to reduce even further the optical absorption of the coating down to subppm level to limit thermal lensing effects (mirror surface deformation induced by the temperature increase).

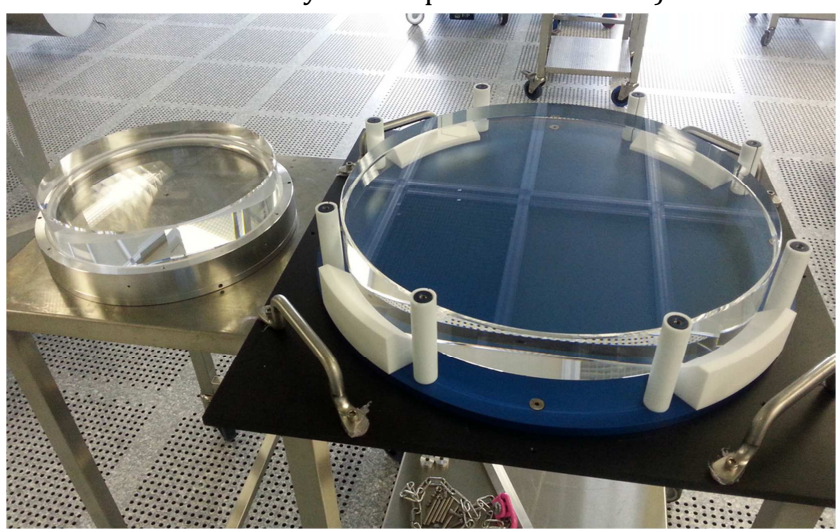

Fig. 1: Advanced Virgo recycling mirror $\emptyset 35 \mathrm{~cm}$ (left) and large Beam Splitter $\emptyset 55$ cm (right)

Moreover, in the advanced detectors, the two FabryPerot cavities (arms of the Michelson interferometer) must be as identical as possible (same mirror transmission, same finesse). Even if our large IBS coater using RF ion sources is very reproducible, it is almost impossible to reach the symmetry specifications for the High Reflective (HR) coating 
transmission, especially for the Input Mirrors (Transmission of $1.4+/-0.1 \%$ at $1064 \mathrm{~nm}$ with $\Delta \mathrm{T}<0.01 \%$ ). The only solution is to coat the two substrates in the same run using a planetary motion. In addition, the transmission value must be uniform on each mirror on a large area implying a strong improvement of the coating uniformity on a large scale (better than $0.1 \%$ over $15 \mathrm{~cm}$ diameter). This last requirement is particularly difficult for the HR coating of the EM whose total thickness is close to $6 \mu \mathrm{m}$.

In the following sections, after having detailed the silica substrate and the coating specifications, we describe how we managed to improve the mirror absorption and the scattering at a level much lower than the first generation of VIRGO mirrors [3]. Secondly, the way to improve the multilayers ( $\mathrm{Ti}^{-} \mathrm{Ta}_{2} \mathrm{O}_{5} / \mathrm{SiO}_{2}$ ) thickness uniformity on a large diameter is detailed.

\section{SILICA SUBSTRATES AND COATING SPECIFICATIONS}

As an example, the substrates (Ø35 cm, $20 \mathrm{~cm}$ thick) chosen for the Advanced Virgo Fabry-Perot cavities mirrors are made of ultra-pure fused silica. It was decided to use the last generation of Suprasil developed by Heraeus (Suprasil 3002) as bulk material for the IM. This choice is mainly driven by the high homogeneity of the refractive index and the extremely low bulk absorption at $1064 \mathrm{~nm}: \mathbf{0 . 2} \mathbf{~ p p m} / \mathbf{c m}$ (part per million). For the EM, crossed by only a small amount of light, the constraints are less stringent and Suprasil 312 was chosen.

Very severe polishing specifications were defined in order to guarantee after coating a low scattering level as well as extremely good flatness (Fig. 2). Indeed, here are below the most stringent specifications asked to the polishing company:

- Microroughness < 1 Angström RMS

- Flatness $<0.5 \mathrm{~nm}$ RMS on the central $\emptyset 15 \mathrm{~cm}$ (low spatial frequencies $<1 \mathrm{~mm}^{-1}$ )

- Point defects : density $<1 / 4 \mathrm{~mm}^{2}$ in $\emptyset 15 \mathrm{~cm}$ (defects< $5 \mu \mathrm{m}),<15$ in $\emptyset 15 \mathrm{~cm}$ (defects [ $5 \mu \mathrm{m}, 50 \mu \mathrm{m}]$ )

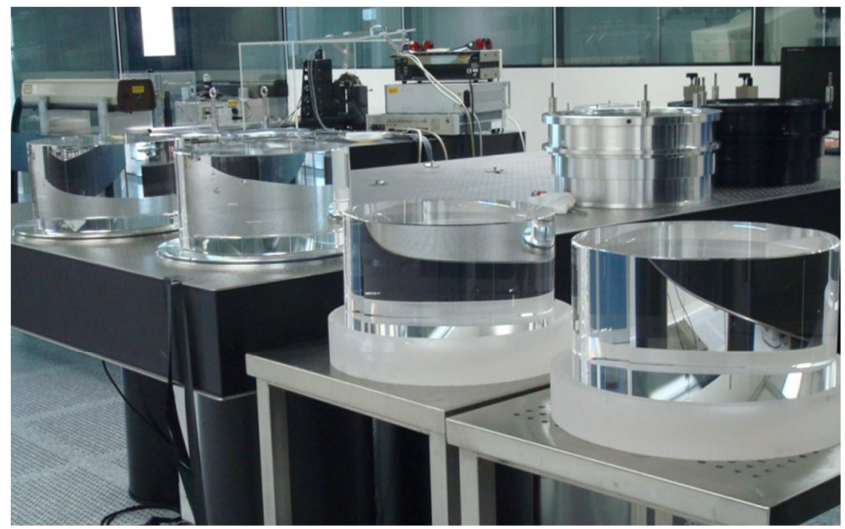

Fig. 2: Four Advanced LIGO ITM substrates before coating

On Fig. 3, a map of the point defects (Micromap profilometer measurement) and a map of the flatness (Fig. 4) on an EM substrate are shown. The optical performances of these substrates are remarkable and better than expected. The measured microroughness is around 0.6 Angström RMS.

Regarding the IBS high reflective coating specifications on the IM and EM, ultimate performances in term of absorption, scattering, flatness are required:
- Flatness after HR coating: $<0.5 \mathrm{~nm}$ RMS on $\emptyset 15 \mathrm{~cm}$ (low spatial frequencies $<1 \mathrm{~mm}^{-1}$ ) with all Zernike terms $<0.5$ nm in amplitude

- Round Trip Losses lower than 50 ppm

- Average absorption at $1064 \mathrm{~nm}<0.5 \mathrm{ppm}$ (part per million)

- Average scattering at $1064 \mathrm{~nm}<10 \mathrm{ppm}$

- HR transmission matching for IM at $1064 \mathrm{~nm}: \Delta \mathrm{T}<$ $0.01 \%$ with $\mathrm{T}=1.4+/-0.1 \%$

- 3 bands (532, 800, $1064 \mathrm{~nm}$ ) AR coating : $\mathrm{R}<100 \mathrm{ppm}$ at $1064 \mathrm{~nm}$ (backside of the mirror)

In addition, a low mechanical dissipation of coating is needed to minimize thermal noise.

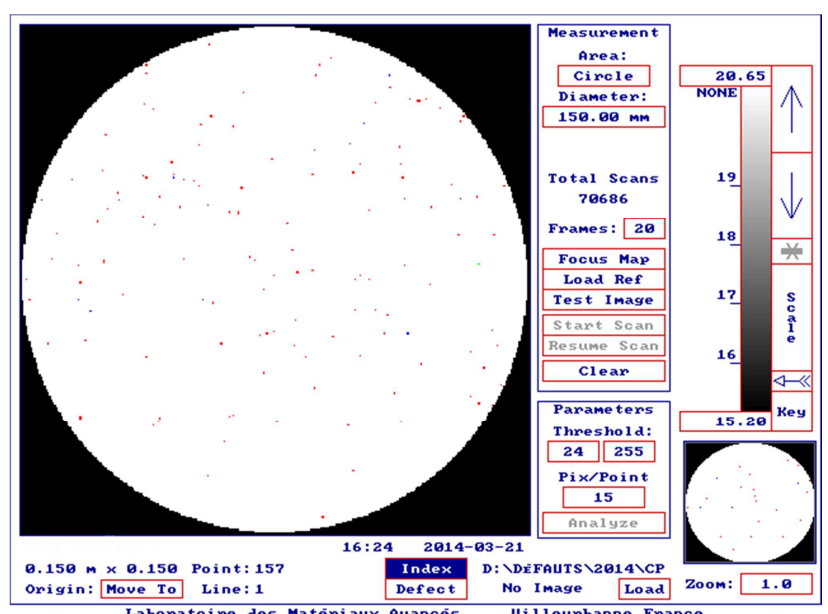

Fig. 3: Point defect map $\emptyset 15 \mathrm{~cm}$ of an Advanced Virgo EM

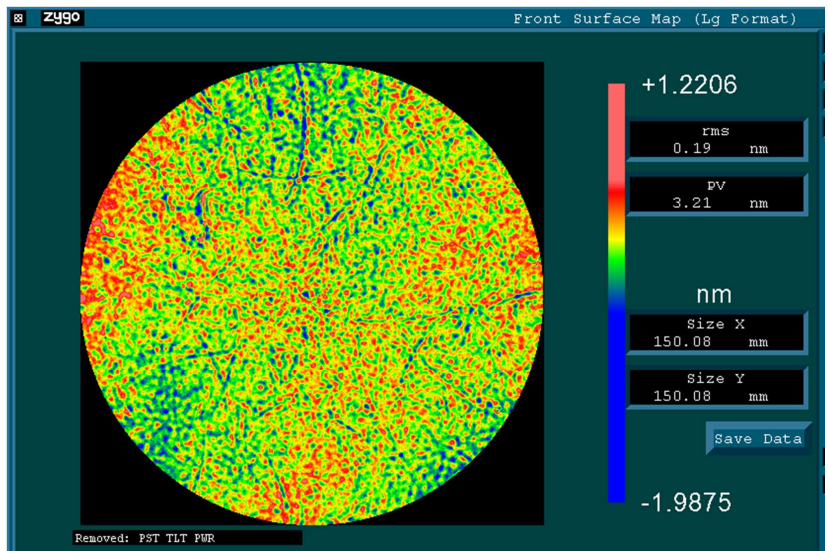

Fig. 4: Surface flatness $(\mathbf{0 . 1 9} \mathbf{~} \mathbf{m}$ RMS $\emptyset 15 \mathbf{~ c m})$ of an Advanced Virgo EM

\section{THE ADVANCED VIRGO AND ADVANCED LIGO INPUT AND END MIRRORS}

\section{A. Thermal noise and absorption}

Ten years ago, one important source of noise in the Virgo interferometer was identified to be the coating thermal noise. A lot of work has been done so far [5] to modify and optimize the high index layers $\left(\mathrm{Ta}_{2} \mathrm{O}_{5}\right)$ which are the main source of mechanical loss (directly proportional to the $\mathrm{Ta}_{2} \mathrm{O}_{5}$ 
total thickness). The best compromise found was to dope Ta205 with Ti atoms.

In order to decrease the high index layer total thickness while keeping the mirror properties, alternative mirror coating designs with non-quarter-wave layers $\mathrm{aH}(\mathrm{bL}$ $\mathrm{cH}) \mathrm{x}$ dL, with $\mathrm{a}, \mathrm{c}<1$ and $\mathrm{b}>1$ ) have been studied [2]. Experimental measurements (TNI or Thermal Noise Interferometer, Caltech USA) proved that the coating mechanical losses decreased [7], compared to a classical design and that the average absorption at $1064 \mathrm{~nm}$ is also lower (from our measurements based on the photothermal deflection technique). We applied this recipe to develop the high reflective coating on the IM and EM of Advanced Virgo.

Fig. 5 shows an absorption map realized on an EM HR coating whose transmission is $3 \mathrm{ppm}$ at $1064 \mathrm{~nm}$. The result is remarkable: average value $\mathbf{0 . 2 4} \mathbf{p p m}$ on $\emptyset 15 \mathbf{~ c m}$ at $1064 \mathrm{~nm}$.

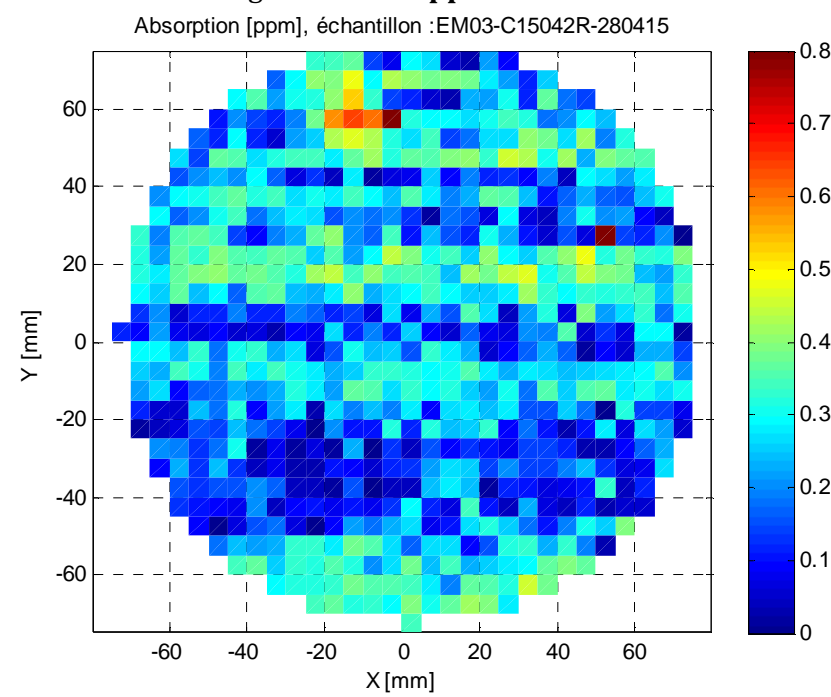

Fig. 5: Absorption map at $1064 \mathrm{~nm}$ on $\varnothing 15 \mathrm{~cm}$ of a high reflectivity coating of an EM of Advanced Virgo

For the 20 Advanced LIGO test masses coated, here is below the average absorption on $\emptyset 15 \mathrm{~cm}$ obtained for the two kinds of HR coating (Input mirrors, End mirrors):

- $\quad$ On the 10 ITMs : $0.22+/-0.03$ ppm (average $+/-\sigma$ )

- On the 10 ETMs : $0.27+/-0.07 \mathrm{ppm}$

\section{B. Scattering}

To improve the average scattering level after coating, a new wet cleaning machine was developed which allows us to have a better cleaning efficiency on the very small particles.

Moreover, a new procedure was put in place to control of the substrate surface just before closing the coating chamber door (visual observation in the dark with a high power halogen lamp, use of ionized dry air gun).

For the 20 Advanced LIGO test masses coated, here is below the average scattering on $\emptyset 15 \mathrm{~cm}$ at $1064 \mathrm{~nm}$ obtained for the two kinds of HR coating (Input mirrors, End mirrors):

- On 10 ITMs : $3.7+/-1.2 \mathrm{ppm}$

- On 10 ETMs : $4.9+/-1.5$ ppm

- $\quad$ Best result : 2.3 ppm (Fig. 6)

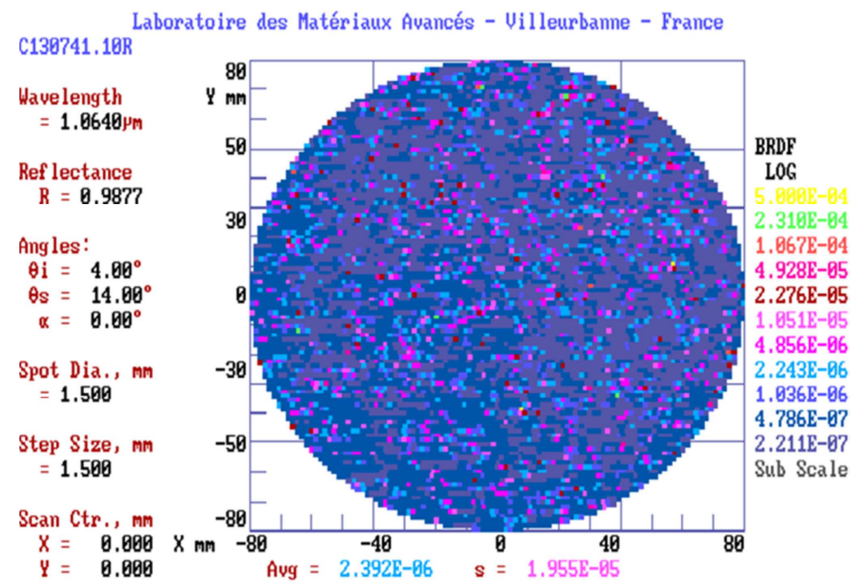

Fig. 6: Average scattering map $\emptyset 15 \mathrm{~cm}$ on a ITM of Advanced LIGO measured with a CASI scatterometer

\section{Antireflective coating}

We developed a specific three band antireflective coating (532, 800 and $1064 \mathrm{~nm}$ ) based on a 16 layers design necessary for the Advanced Virgo interferometer.

Due to the stringent specification at $1064 \mathrm{~nm}(\mathrm{R}<100 \mathrm{ppm}$ with a goal of $50 \mathrm{ppm}$ ), we tried to find during the design optimization a solution for this wavelength as less sensitive as possible to manufacturing errors. We have done 1000 situations to test the robustness of the design by applying a random error of $+/-1 \%$ on all layers. On Fig 7 , we can see the worst trial and the higher reflectivity value we can have is 100 ppm.

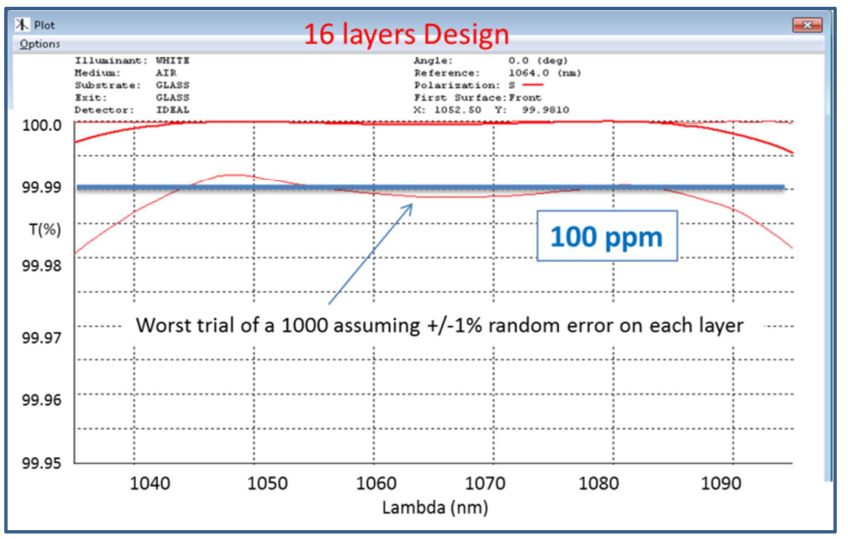

Fig. 7: Zoom of the AR spectrum around $1064 \mathrm{~nm}$ with sensitivity to errors (of random manufacturing errors $+/-1 \%$ on all layers)

The design found has shown experimentally a remarkable robustness to errors. Indeed, $100 \%$ of AR coatings made on large optics using this design on more than 20 large substrates have a reflectivity lower than 100 ppm, $80 \%$ less than $50 \mathrm{ppm}$. An example of achievement can be seen on Fig. 8: $13 \mathrm{ppm}+/-6 \mathrm{ppm}$ on $\emptyset 15 \mathrm{~cm}$. 


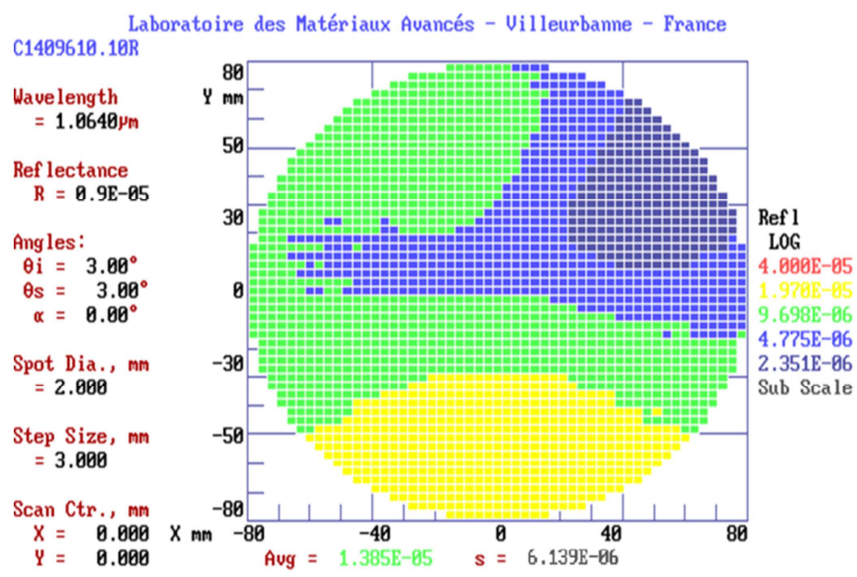

Fig. 8: Reflexion map at $1064 \mathrm{~nm}$ on $\emptyset 16 \mathrm{~cm}$ of an AR coating of an ITM of Advanced LIGO (average value $13 \mathrm{ppm}$ )

\section{Mirror Flatness}

For the first generation of Virgo mirrors made in 2002 and 2009, a large IBS (Ion Beam Sputtering) coating chamber was developed at LMA (Fig. 9). The coating thickness uniformity needed was not so stringent: indeed the mirror flatness should be lower than $3 \mathrm{~nm}$ RMS on $\emptyset 15 \mathrm{~cm}$. For the Advanced detector, the flatness must be better than $0.5 \mathrm{~nm}$ RMS over the same diameter: this is a huge difference.

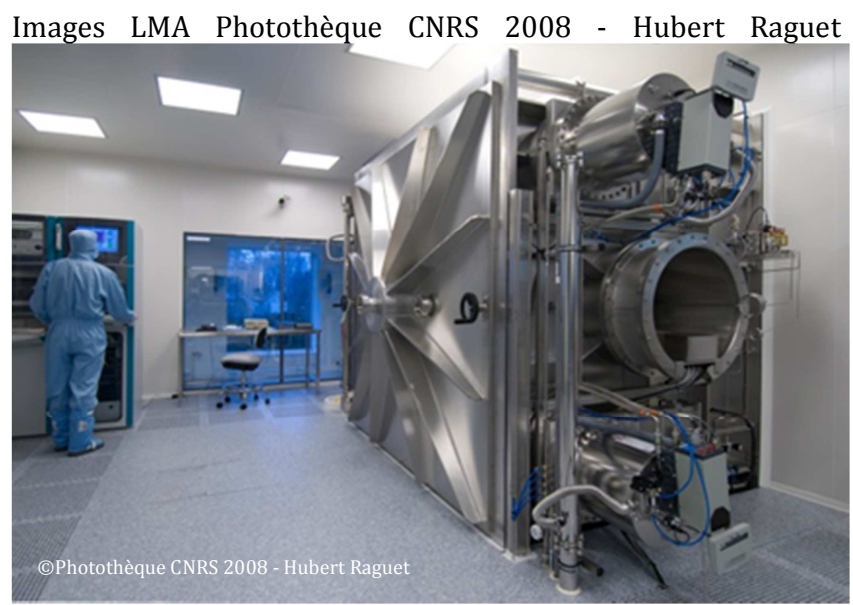

Fig. 9: Large IBS coater

Coating thickness control is a crucial point for interference coatings. In the Advanced gravitational waves interferometers, it is required to have coatings with very good thickness uniformity in order to provide constant optical properties over the optics surface. Two Fabry-Perot cavities (made of one Input and one End mirror) must have the same optical characteristics: mirror transmission, finesse, round trip losses. It implies to coat two $35 \mathrm{~cm}$ silica substrates (Fig. 9) at the same time (twin mirrors). Indeed, it is unthinkable to get the same mirror transmission at the level required in two successive runs, even if our IBS coater is very reproducible.

To reach the flatness requirement after coating on the Advanced LIGO and Advanced Virgo large mirrors, we developed at LMA a new sample holder with a planetary motion coupled with dedicated masks (between the targets and the substrates).

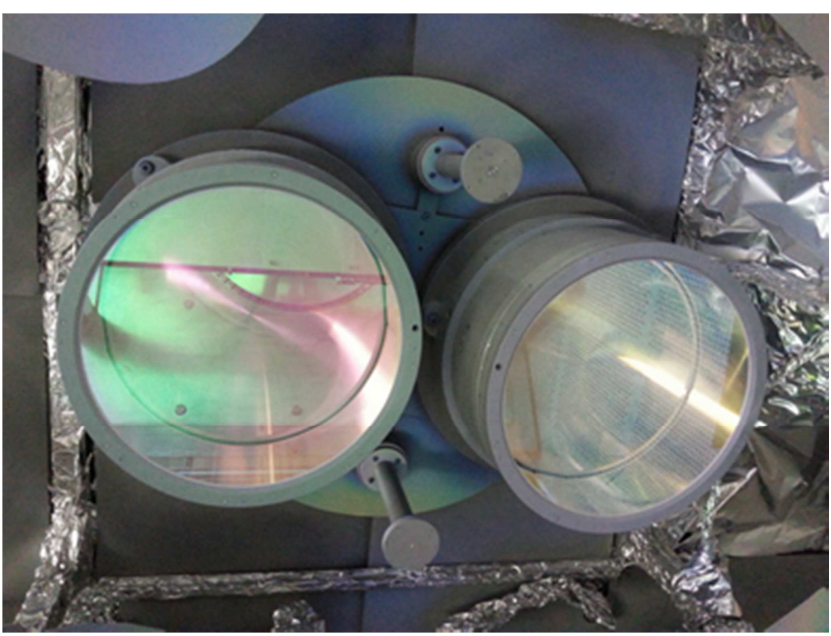

Fig. 10: Two $\emptyset 35 \mathrm{~cm}$ IM installed in the coater sample holder

For each material, a mask shape is calculated with an homemade simulation software because the sputtered particles profiles are different. Thus, we are able to coat two $40 \mathrm{~kg}$ mirrors in the same run (Fig. 10).

The mask shape optimization was made first on monolayer deposited on large fused silica plates (Ø35 cm, $6 \mathrm{~mm}$ thick) with spectrophotometric measurements along diameters.

Some limitations linked to the metrology device were rapidly observed when the uniformity level became lower than $0.2 \%$ on $\emptyset 16 \mathrm{~cm}$ (unable to well optimize the mask shape, uncertainties).

So we decided to measure the coating uniformity directly on the HR coatings. The uniformity profiles are deducted from reflectivity measurements at large AOI on the edge of the reflectivity band of the multilayer using a CASI scatterometer. The reflectivity variation are converted in centering variation and then in thickness variation.

Fig. 11 shows the average uniformity profile obtained on $\emptyset 24$ $\mathrm{cm}$ for a HR coating of an EM ( $6 \mu \mathrm{n}$ total thickness). The uniformity reached is remarkable: \#0.05\% on $\emptyset 20 \mathbf{c m}$ or $3 \mathrm{~nm}$ PV for a HR coating of an EM.

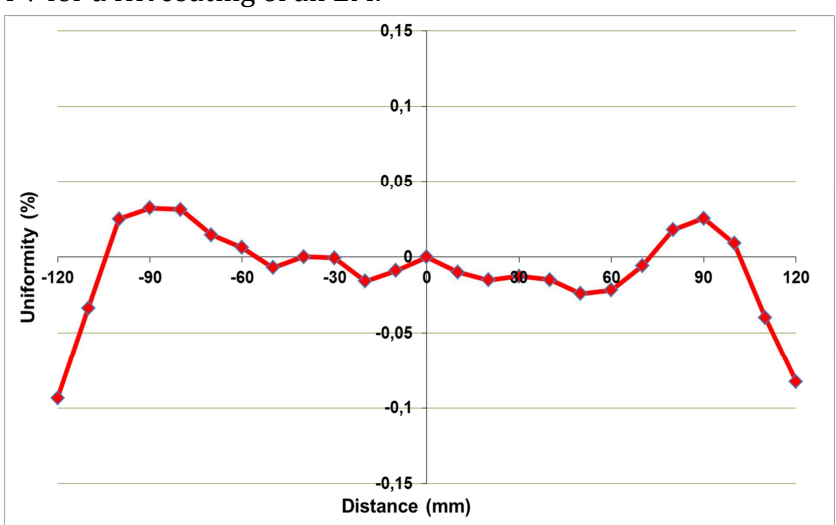

Fig. 11: Average uniformity profile on $\emptyset 24 \mathrm{~cm}$ of an EM high reflective coating

Fig. 12 shows the flatness achieved on a HR coating of an Advanced Virgo EM (total thickness $6 \mu \mathrm{m}$ ) which satisfies the requirement: $0.37 \mathbf{~ n m}$ RMS on $\emptyset 15 \mathbf{~ c m}$. 
The transmission matching of the two IM and the two EM was also a success (average transmission difference between the two IM is $0.002 \%$ ).

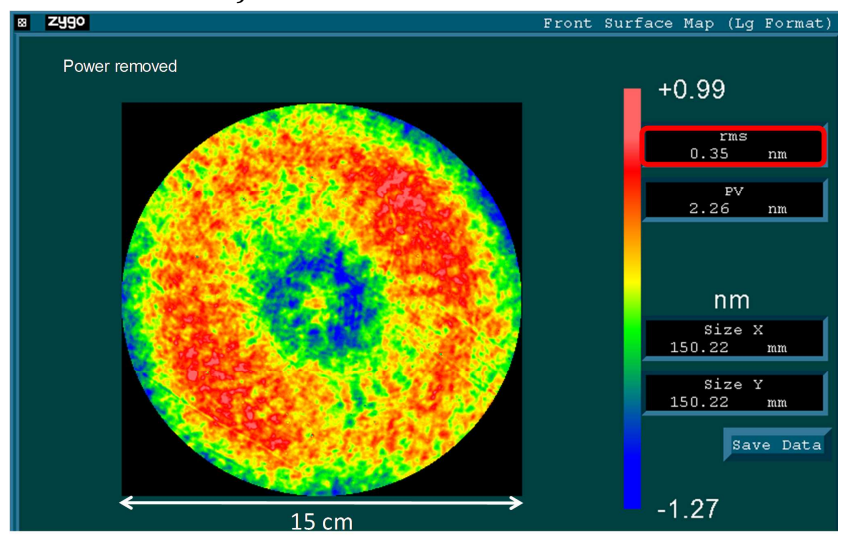

Fig. 12: Flatness of an Advanced Virgo EM on $\emptyset 15 \mathrm{~cm}$ (Power removed)

\section{CONCLUSION}

LMA was able to reach the required specifications for the arm cavity mirrors of the advanced gravitational wave detectors. This is the first time such performances on coating uniformity, absorption, scattering were achieved on $35 \mathrm{~cm}$ mirrors made by IBS.

We can in any case say that the first detected gravitational wave (and the future ones....) has passed between two large mirrors coated at LMA.

\section{References}

1. B. P. Abbott et al., "Observation of Gravitational Waves from a Binary Black Hole Merger", Physical Review Letters, 116, 061102 (2016).

2. L. Pinard, B. Sassolas, R. Flaminio, D. Forest, A. Lacoudre, C. Michel, J.L. Montorio, N. Morgado, Optics Letters, "Toward a new generation of low-loss mirrors for the advanced gravitational waves interferometers", Optics Letter vol. $36 n^{\circ} 8$ (2011) 1407

3. B. Cimma, D. Forest, P. Ganau, B. Lagrange, J.M. Mackowski, C. Michel, J.L. Montorio, N. Morgado, R. Pignard, L. Pinard, A.Remillieux, "IBS sputtering coatings on large substrates: towards an improvement of the mechanical and optical performances", Applied Optics, vol. $45 n^{\circ} 7$ (2006) 1436-1439

4. R. Bonnand - "The Advanced Virgo gravitational waves detector : Study of the optical design and development of the mirrors" - PhD : https://tel.archives-ouvertes.fr/tel-00980687

5. M. Granata, E. Saracco, N. Morgado, A. Cajgfinger, G. Cagnoli, J. Degallaix, V. Dolique, D. Forest, J. Franc, C. Michel, L. Pinard, R. Flaminio, "Mechanical loss in state-of-the-art amorphous optical coatings", Physical Review D 93, 012007 (2016)

6. B. Sassolas, R. Flaminio, J. Franc, C. Michel, J.L. Montorio, N. Morgado, L. Pinard, "Masking technique for coating thickness control on large and strongly aspherical optics", Applied Optics, vol.48 n¹9 (2009) 3760

7. A. E. Villar et al., Measurement of thermal noise in multilayer coatings with optimized layer thickness, Phys. Rev. D 81, 122001 (2010) 